\title{
LA REFORESTACIÓN DEL ÁREA DE USO PÚBLICO Y LOS EFECTOS DEL ARCO SECO DE AZUERO
}

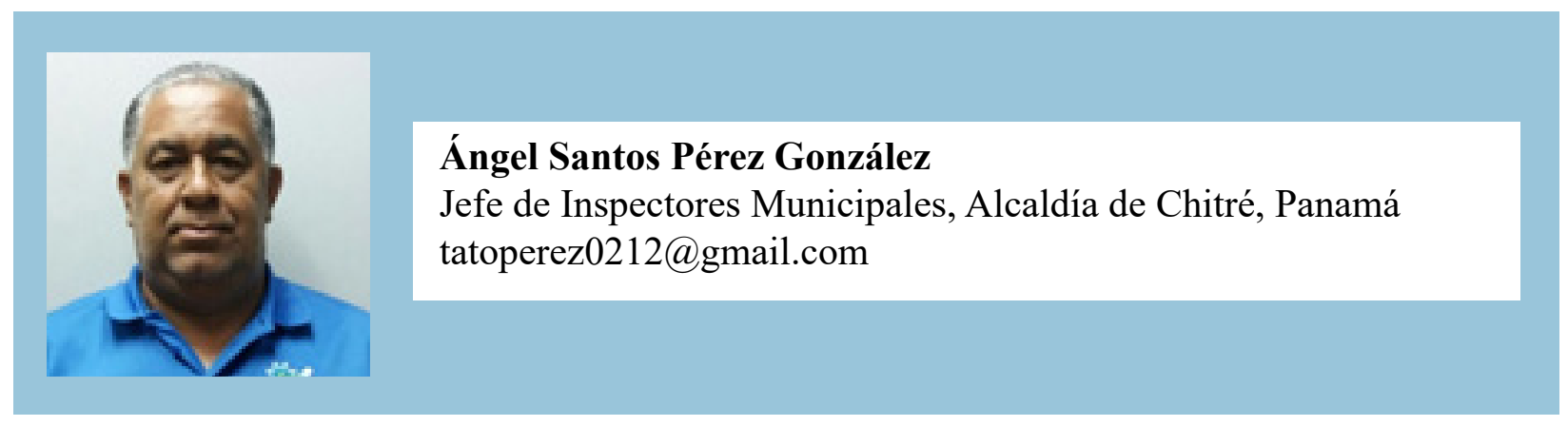

\section{RESUMEN}

Los espacios de uso público son todos aquellos que el urbanizador o inversionista cede gratuitamente al estado para los sistemas viales, de acueducto, sanitario, pluvial y todos aquellos utilizados como aceras, parques, áreas verdes, jardines, áreas deportivas, que bien utilizadas pude contribuir a mitigar los efectos del arco seco de Azuero. Este artículo pretende sensibilizar a la población del corregimiento de Monagrillo sobre la importancia de la reforestación de estas áreas de uso público y demostrar que los costos para la adquisición, plantación y mantenimiento de los árboles que se puedan sembrar en los espacios de uso público son mínimos ante los beneficios que puede brindar al medio ambiente. La metodología utilizada para la redacción del artículo es de tipo no experimental, transaccional, con un estudio explicativo, con hipótesis causal; el universo es la población del corregimiento de Monagrillo y la muestra son cinco urbanizaciones, escogidas al azar; el tipo de muestreo es el simple al azar; y los sujetos son las personas encuestadas. Para el análisis de los datos se empleará encuesta y porcentajes. Como parte de los resultados, se dieron a conocer los costos de adquirir, plantar y mantener árboles en el área de uso público de las cinco urbanizaciones escogidas al azar del corregimiento de Monagrillo.

Palabras Clave: Reforestación, área de uso público, Arco Seco de Azuero. 


\title{
THE REFORESTATION OF THE AREA OF PUBLIC USE AND THE EFFECTS OF THE AZUERO DRY ARCH
}

\begin{abstract}
The spaces for public use are all those that the developer or investor gives free to the state for road systems, aqueduct, sanitary, rain and all those used as sidewalks, parks, green areas, gardens, sports areas, well used could contribute to mitigate the effects of the dry arc of Azuero. This article aims to raise awareness among the population of the village of Monagrillo about the importance of reforestation of these areas for public use and to demonstrate that the costs for the acquisition, planting and maintenance of trees that can be planted in public spaces are minimal before the benefits it can provide to the environment. The methodology used for writing the article is nonexperimental, transactional, with an explanatory study, with a causal hypothesis; the universe is the population of the Corregimiento of Monagrillo and the sample is five urbanizations, chosen at random; the type of sampling is simple random; and the subjects are the people surveyed. For the analysis of the data, a survey and percentages will be used. As part of the results, the costs of acquiring, planting and maintaining trees in the area of public use of the five urbanizations chosen at random from the village of Monagrillo were announced.
\end{abstract}

Key words: Reforestation, Public use area, Azuero dry arch.

\section{INTRODUCCIÓN}

La deforestación más que un problema ambiental es un problema social y se vuelve tan complejo debido a que es un efecto de actividades humanas en las que prevalecen intereses de toda índole desde los más grandes por parte de ciudadanos acaudalados y/o funcionarios con el rango más alto en el Estado, hasta el campesino más humilde que requiere de un espacio libre de árboles para el establecimiento de su parcela de cultivo.

La superficie cubierta de bosque en el país cada año es menor y para que esto ocurra se conjugan una serie de elementos que es muy difícil ordenar según su importancia ya que todos tienen su cuota influencia en este fenómeno. Podemos empezar con el Estado mismo; quien debería promover políticas en función de un país eminentemente forestal y aquí es donde entran en los intereses curiosamente dentro de la misma Administración Pública. 
Alrededor del mundo se hace concientización de lo peligroso que son las quemas y tala de bosques, pero al parecer debido a la gran sobrepoblación que es directamente proporcional al aumento de las urbanizaciones en el corregimiento de Monagrillo y en todo el mundo ya sea países desarrollados o en vías de desarrollo - por este motivo es casi imposible evitarlo en algunos lugares que aún se conservan reservas forestales que son las que contribuyen en gran parte a mantener el clima un poco estable.

Las urbanizaciones vienen a cubrir el déficit habitacional en nuestro corregimiento y con esto los problemas que conllevan a la hora de obtener los permisos de lotificación, estudios de impacto ambiental, permisos de construcción y ocupación, etc.

La deforestación es motivada por varias causas, la mayoría de las cuales se originan fuera ecológica, social, económica, cultural y política en una región dada.

Las causas de la deforestación pueden separarse en dos categorías. La primera implica aquellos factores ligados directamente al acto de tala de árboles o degradación de la tierra, denominadas como causas directas o próximas. La segunda categoría incluye factores sociales de fondo que generan las causas directas, a las que se denominan causas subyacentes.

La deforestación es el producto de la interacción de numerosas fuerzas ecológicas, sociales, económicas, culturales y políticas en una región dada. Hoy en día se reconoce ampliamente que los obstáculos más grandes para combatir la deforestación y la degradación de los bosques provienen de los sectores externos al sector forestal.

En el corregimiento de Monagrillo se encuentra en problemática, que está ocurriendo a nivel mundial, la deforestación, es un proceso generalmente provocado por la acción humana. La creciente llegada de personas que abandonan el área rural, la campiña panameña y el aumento de la población en forma exponencial, hacen que surja la necesidad de creación de nuevas, modernas y más seguras urbanizaciones en nuestro querido corregimiento.

Estas urbanizaciones vienen a cubrir la creciente necesidad del déficit habitacional que existe en este y otros corregimientos del Distrito de Chitré. Como dice el dicho: "Chitré, la ciudad que crece sola" y "En Chitré nadie es forastero ", son dos frases que por si decir, aumenta la población del corregimiento de Monagrillo, por eso podemos sacar la conclusión que el aumento de la población 
es directamente proporcional al aumento en este sector.

Con la creación de nuevas urbanizaciones aumenta, considerablemente el grado de deforestación. Esto es algo normal en estos menesteres, porque obligatoriamente deben de talarse árboles para las construcciones de nuevas urbanizaciones y así cubrir una necesidad básica que es la vivienda. En este estudio investigativo daremos a conocer una posible solución en cuanto a la deforestación y al mismo tiempo en las construcciones de nuevas urbanizaciones. Según el Ministerio de Vivienda y Ordenamiento Territorial, Decreto Ejecutivo No 36 de 31 de agosto de 1998, "Por el cual se aprueba el Reglamento Nacional de Urbanizaciones, de Aplicación en el Territorio de la República de Panamá", Capítulo III, De las Cesiones Obligatorias en su artículo 42 expresa lo siguiente: "Las superficies destinadas para áreas verdes o recreativas a que se hace referencia en los artículos anteriores, tendrán los siguientes porcentajes:

a. Urbanizaciones de baja densidad de población: de 25 pers. /ha. a 300 pers. /ha. Zonificación R-R, R1-A, R1-B, R2-A y R2-B 7.5\% del área útil de lotes.

b. Urbanizaciones de mediana densidad de población. De 400 pers. /ha. a 600 personas/ha Zonificación R-3, R-E y RM 10\% del área útil de lotes.

c. Urbanizaciones de alta densidad de población. de 750 a 1500 pers. /ha. Zonificación RM-I, RM-2, RM-3. 15\% del área útil de lotes.

d. Cuando en el uso comercial urbano (C-2) se proyecta la aplicación del uso residencial se computará con la densidad más alta circundante en un radio de $500 \mathrm{mts}$. al proyecto. En caso de que se demuestre que el uso comercial (C-2) será utilizado únicamente como tal, el área a ceder para uso públicos será de $7.5 \%$ del área útil de lotes.

e. Urbanizaciones industriales: Uso de Suelo Industrial (I) 7.5\% del área útil de lotes”.

Para el corregimiento de Monagrillo las urbanizaciones de mediana densidad de población es donde recae todas las urbanizaciones de Monagrillo y de allí este 10\% está destinado a las áreas de uso público, que no es más que los espacios urbanos, o de periferia a éstos, predominantemente ocupados con árboles, arbustos o plantas, que pueden tener diferentes usos, ya sea cumplir funciones de esparcimiento, recreación, ecológicas, ornamentación, protección, recuperación y rehabilitación del entorno o similares.

Para este $10 \%$ destinado al área social tendremos como objetivo general adquirir, plantar y mantener árboles en el área de uso público de las urbanizaciones del corregimiento de Monagrillo. 


\section{METODOLOGÍA}

Se tomará una muestra representativa, mediante una encuesta, con preguntas abiertas, aplicadas a una muestra de la población del corregimiento de Monagrillo (población finita), con el propósito de hacer el análisis de la información. El diseño es no experimental que no permite identificar y cuantificar las causas de un efecto dentro de este estudio se complementa con el de tipo transaccional que permite la recolección de datos en un solo momento, en un tiempo único y su propósito es describir variables, y analizar su incidencia e interrelación en un momento dado. Ambos buscan encontrar las razones o causas que ocasionan ciertos fenómenos, su objetivo es explicar por qué ocurre un fenómeno y en qué condiciones se da este. Asimismo con una hipótesis causal se pretende identificar y analizar las causas, variables independientes, y sus resultados, los que se expresan en hechos verificables, variables dependientes); el universo es la población del corregimiento de Monagrillo en donde la muestra son cinco urbanizaciones Laura Antonia, Villa Lineth I, Villa Dorada, El Encanto y Villa San Miguel, escogidas en un muestreo simple al azar en donde se aplican encuestas estratificada por porcentajes.

A continuación se presentan los resultados más relevantes del estudio:

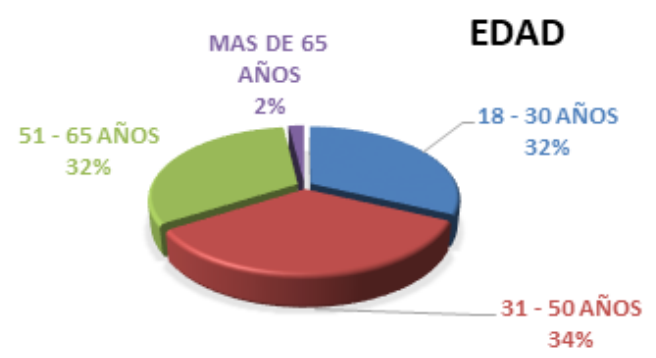

La edad para estudio es muy importante, a la hora de tomar conciencia en favor de los problemas ambientales existentes en nuestro querido corregimiento y a nivel mundial. El estudio arrojó que entre 18 - 30 años fueron encuestados 16 personas, entre $31-50$ años fueron encuestados 17 personas, entre $51-65$ años fueron encuestados 16 personas y más de 65 años fue encuestado una persona.

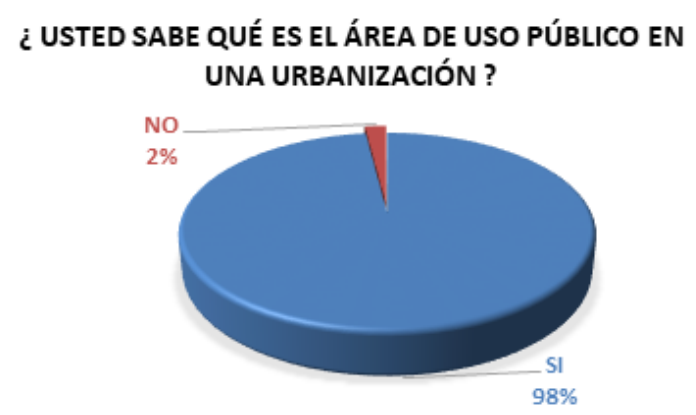


El nivel de escolaridad es sumamente importante para este estudio, entre mayor escolaridad más consciente se es ante los problemas ambientales. El estudio arrojó que sin escolaridad no se encuesto a ninguna persona, de primaria solo se encuesto a 2 personas, de secundaria se encuesto a 14 personas y de universidad se encuesto a 34 personas.

El conocimiento sobre el área de uso público a los habitantes de las cinco urbanizaciones escogidas al azar en cuanto a la concientización sobre los problemas ambientales será decisivo a la hora de la realización de esta encuesta. El estudio arrojo que 49 personas encuestadas tenían conocimientos y una sola persona no tenía conocimiento.

\section{¿ USTED SABE QUÉ ES LA REFORESTACIÓN ?}

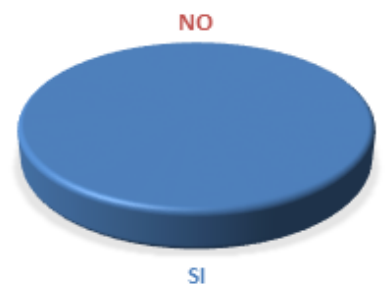

Como sabemos la reforestación debe de ser un problema para todos los habitantes del planeta tierra y así tener un futuro con menos problemas ambientales. El estudio arrojó que el 100 \% o sea que las 50 encuestados sabía qué es la reforestación.

Afortunadamente todo el mundo hoy en día habla sobre los beneficios de plantar un árbol como una actividad para ayudar al planeta, contribuir con la generación de oxígeno y así dejar tu granito de arena.

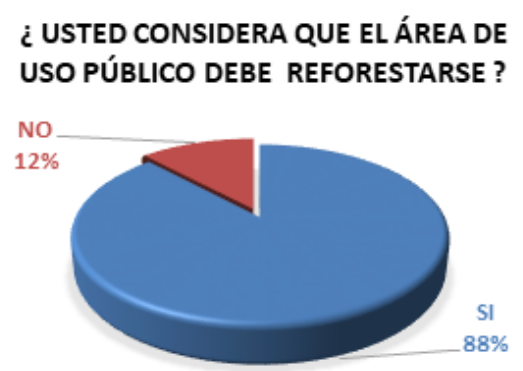

El costo total del proyecto de inversión ambiental es de B/. 7953.40, esta inversión no es nada en comparación con los efectos positivos que dará al medio ambiente que rodea las Urbanizaciones 
de Monagrillo. El estudio arrojó que 44 encuestados si quieren que se reforeste esta área y 6 personas no quieren se reforeste.

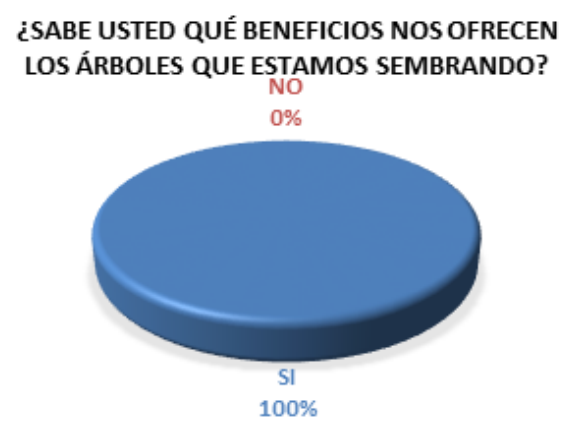

Los árboles absorben los olores y gases contaminantes (óxidos de nitrógeno, amoníaco, dióxido de azufre y ozono) y filtran las partículas contaminantes del aire, atrapándolas en sus hojas y corteza. El estudio arrojó que el 100 \% ósea que las 50 personas encuestadas saben los beneficios que nos ofrecen los árboles que se van a sembrar en las urbanizaciones escogidas al azar.

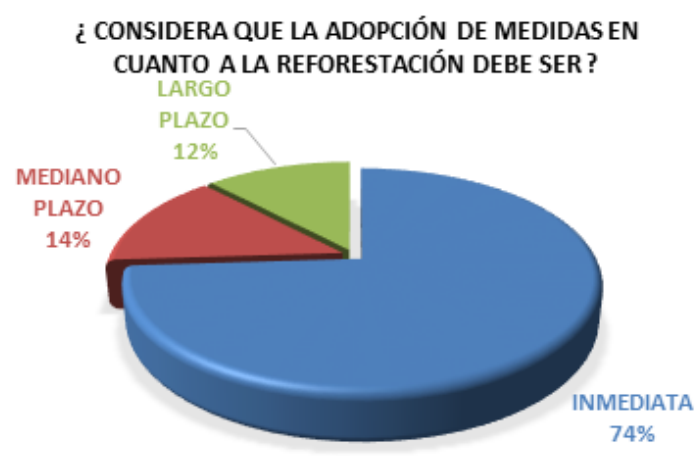

Conocer las soluciones para la deforestación puede ayudar a reducir en gran medida este problema, ya que con esto nos estaremos dando cuenta de las posibles medidas que podemos tomar para colaborar y contribuir a que los árboles y los bosques se mantengan siempre verdes y conservados. El estudio arrojó que 37 personas encuestadas consideraban que se deberían tomar medidas inmediatas que son necesarias en cuanto a la reforestación.

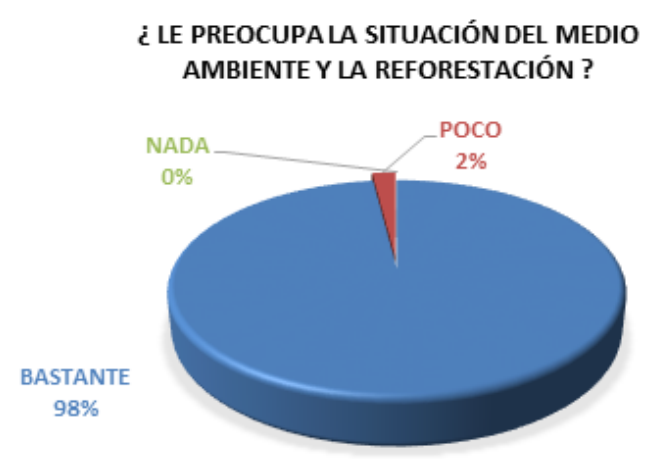


Luego de la catástrofe ambiental que han vivido las regiones del país que han sido afectadas por los incendios forestales, comienza a planificarse la reforestación de los terrenos arrasados. El estudio arrojó que 49 personas encuestadas le preocupa la situación del medio ambiente, la reforestación y solo una persona no le preocupa.

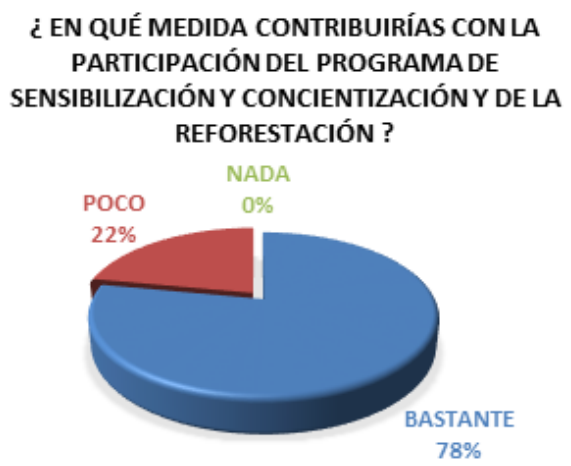

La realización de actividades de reforestación en el entorno escolar tiene como principal objetivo implicar a la Comunidad Educativa en las tareas de defensa, conservación y recuperación de los bosques, fomentando su estudio y conocimiento. Más allá de la propia reforestación, se intenta crear una relación afectiva con los árboles plantados mediante tareas de mantenimiento y observación. La realización de la plantación actúa como eje en torno al cual articular una importante cantidad de contenidos relacionados con la preservación de nuestro entorno. El estudio arrojó que 38 personas encuestadas contribuirían con la participación del programa de sensibilización y concientización y de la reforestación y 11 personas no estarían de acuerdo con medidas que se adopten.

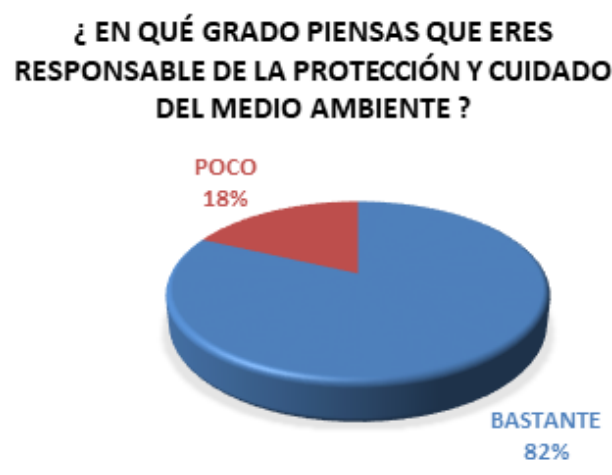

La responsabilidad ambiental recae tanto en los individuos, como en las empresas, países y en la especie humana en su conjunto. El estudio arrojó que 41 personas encuestadas piensan que son responsables de la protección y cuidado del medio ambiente y 9 personas piensan que no son responsables. 


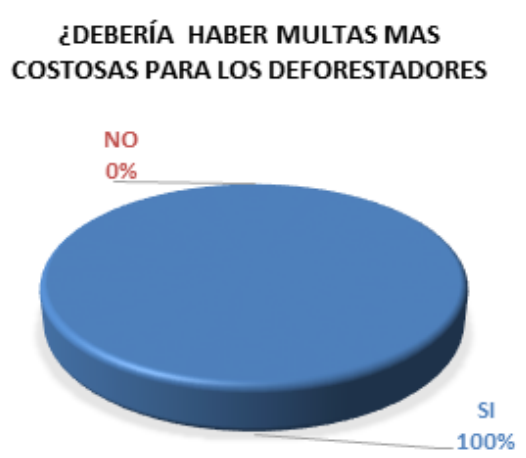

De ahora en adelante el que deforeste tendrá que pagar. El principal propósito, es que se reparen los daños causados a través de multas. Otra parte de esos recursos deberán ser utilizados en programas de reparación del ecosistema y "servir de estímulo para que el causante mejore su gestión ambiental". El estudio arrojó que el 100 \% en donde 50 personas encuestadas que deberían de multas muchas más severas para los deforestadores.

Como lo expresamos anteriormente, para la encuesta, escogimos cinco urbanizaciones al azar en el corregimiento de Monagrillo (Laura Antonia, Villa Lineth, Villa Dorada, El Encanto y Villa San Miguel), con el método transaccional escogimos las calles y los hogares (10 por cada urbanización) donde realizamos dicha encuesta. Según el experto en suelo y tipos de árboles Amado Atencio Técnico Colaborador de Mi Ambiente en el Distrito de Ocú, los árboles recomendados para la calidad del suelo del corregimiento de Monagrillo son: guayacanes y robles. En la tabla siguiente daremos a conocer los costos de adquirir, plantar y mantener árboles en el área de uso público.

\begin{tabular}{|c|c|c|c|c|c|c|c|c|c|c|}
\hline \multicolumn{11}{|c|}{ TABLA DE COSTOS DE ADQURRR, PLANTAR Y MANTENER ARBOLES EN EL AREADE USO PUBLICO } \\
\hline \multirow{2}{*}{$\mathrm{N}^{\circ}$} & \multirow{2}{*}{ URBANZACON } & M2DE AREA DE & TOTALDE & ARBOLES DE & \multirow{2}{*}{ c/us } & \multirow{2}{*}{ совто } & \multirow{2}{*}{$\begin{array}{c}\text { ARBOAESDE } \\
\text { ROBLES }\end{array}$} & \multirow{2}{*}{ c/us } & \multirow{2}{*}{ costo } & \multirow{2}{*}{$\begin{array}{l}\text { COSTO } \\
\text { TOTAL }\end{array}$} \\
\hline & & USO PUBUICO & ARBOAES & GUAYACAN & & & & & & \\
\hline 1 & LAURAANTONIA & $80000 \mathrm{N2}$ & 32 & 16 & B. 0.75 & B. 12.00 & 16 & B. 0.60 & B. 9.60 & 2160 \\
\hline 2 & VLLALNETHI & $1000.00 \mathrm{~N} 2$ & 40 & 20 & B. 0.75 & B. 15.00 & 20 & B. 0.60 & B/. 12.00 & B. $\quad 27.00$ \\
\hline 3 & VILADORADA & $1485.00 \mathrm{M} 2$ & 44 & 2 & B. 0.75 & B. 16.50 & 2 & B. 0.60 & B/. 13.20 & B. \\
\hline 4 & ELENCANTO & $68208 \mathrm{ML}$ & 32 & 16 & B. 0.75 & B. 12.00 & 16 & B. 0.60 & B. 9.60 & B. $\quad 2160$ \\
\hline 5 & VLLA SANMGGEL & $613.55 \mathrm{M} 2$ & 20 & 10 & B. 0.75 & B. 7.50 & 10 & B. 0.60 & B. 6.00 & B. $\quad 13.50$ \\
\hline & COSTO DE PLANTAR & $\mathrm{X}$ & $\mathrm{X}$ & $\mathrm{X}$ & $\mathrm{X}$ & $\mathrm{X}$ & $\mathrm{X}$ & $\mathrm{X}$ & $\mathrm{X}$ & B. $\quad 340,00$ \\
\hline & COSTO DEMANTENIMENTO & $\mathrm{X}$ & $\mathrm{X}$ & $\mathrm{X}$ & $\mathrm{X}$ & $\mathrm{X}$ & $\mathrm{X}$ & $\mathrm{X}$ & $\mathrm{X}$ & B. $\quad 7,500.00$ \\
\hline & TOTAL & $\mathrm{x}$ & 168 & 84 & $\mathrm{x}$ & B/. 63.00 & 84 & $\mathrm{X}$ & B. 50.40 & B. $\quad 7,95340$ \\
\hline & & & & & & & & & & \\
\hline
\end{tabular}




\section{CONCLUSIÓN}

Estos datos indican que la reforestación del área de uso público en la Urbanización de Monagrillo podría amortiguar en cierta medida los efectos del Arco Seco de Azuero, ya que el $88 \%$ de los encuestados sugieren que si se deben reforestar estas áreas.

Como hemos visto, en la tabla anterior, el costo total del proyecto de inversión ambiental es de B/. 7953.40, por lo que esta inversión no es significativamente alta en comparación con los efectos positivos que dará al medio ambiente que rodea las urbanizaciones de Monagrillo. Las plantaciones y la reforestación de las tierras deterioradas y los proyectos sociales de siembra de árboles producen resultados positivos que los bienes que se producen y por los servicios ambientales que prestan.

También esta reforestación impulsa la acción ciudadana en defensa del medio ambiente, participando en acciones forestales, sensibilizando a la población, incentivando la participación social y promueve la asociación de voluntariado ambiental, centros educativos, ayuntamientos, etc. con el objetivo de mejorar, restaurar y conservar espacios naturales degradados, educación ambiental. 


\section{BIBLIOGRAFÍA}

1. Camarena, C. (31 de Enero de 2009). La deforestación es la causa principal de las inundaciones. Obtenido de Panamá América: https://www.panamaamerica.com.pa/mundo/la-deforestaciones-la-causa-principal-de-las-inundaciones-327581

2. Ceuta, L. V. (2015). Línea Verde Ceuta. Obtenido de Reforestación: http://www. lineaverdeceutatrace.com/lv/consejos-ambientales/reforestemos/que-es-la-reforestacion.asp

3. INEC. (2017). Instituto Nacional de Estadística y Censo. Obtenido de Panamá en cifras: https://www.inec.gob.pa/Publicaciones/Default2.aspx?ID_CATEGORIA=17\&ID_ SUBCATEGORIA $=45$

4. MUPA. (1998). REGLAMENTO NACIONAL DE URBANIZACIONES. Obtenido de https://dpu.mupa.gob.pa/wp-content/uploads/2017/06/REGLAMENTO-NACIONAL-DEURBANIZACIONES.pdf 